\title{
Sudden-onset anisocoria in a patient with upper respiratory tract infection
}

\author{
Julio C. Furlan MD PhD, Arun N.E. Sundaram MD MSC
}

A 33-year-old woman presented after sudden onset of a right-sided throbbing, severe headache and drooping of her right upper eyelid while recovering from a 5-day upper respiratory tract infection with persistent dry cough. She had no history of neck trauma or manipulation (e.g., massage or chiropractic therapy). Her previous medical history was unremarkable. She was a smoker (10 cigarettes per day on average), drank a glass of wine or beer a week on average and did not use recreational drugs. She was not taking any prescription drugs.

The findings of her physical and neurologic examinations were normal except for anisocoria (unequal pupil size) and her right upper eyelid being $2 \mathrm{~mm}$ lower than the left. In ambient light, the right pupil was $3 \mathrm{~mm}$ in diameter and the left pupil $4 \mathrm{~mm}$. In dim light, the right pupil expanded to $4 \mathrm{~mm}$, whereas the left pupil measured $6 \mathrm{~mm}$. The right pupil dilated more slowly in the darkness than the left pupil. The patient's extraocular movements were normal, and she had no diplopia in any cardinal position of gaze. There was no clinical evidence suggesting a connective tissue disorder.

\section{What is the cause of the patient's anisocoria?}

a. Right-sided Horner syndrome

b. Paresis of the right third cranial (oculomotor) nerve

c. Physiologic anisocoria

d. Left-sided tonic pupil

The coexistence of miosis (constricted pupil) and ptosis on the same side suggests Horner syndrome (a). On physical examination, the patient had no anhidrosis, which suggests a lesion in the postganglionic oculosympathetic pathway. The degree of anisocoria was increased in darkness (pupillary dilatation lag), which is consistent with sympathetic denervation. Box 1 describes the test we used to show pupillary dilation lag. ${ }^{1}$
Another important differential diagnosis in this case of unilateral headache with ptosis and anisocoria is (b) paresis of the right third cranial nerve (more specifically, third nerve fascicle syndrome). The most common cause of nontraumatic isolated oculomotor nerve paresis with pupillary involvement is an aneurysm of the posterior communicating artery adjacent to its junction with the internal carotid artery. Our patient's history of severe rightsided headache was consistent with an ipsilateral aneurysm of the posterior communicating artery. However, paresis of the right third cranial nerve would cause ipsilateral mydriasis (dilated pupil) and ptosis; in our patient, ptosis was noticed on the right, but the left pupil was larger than the right one (the reverse of the expected in a right third cranial nerve paresis). Furthermore, paresis of the third cranial nerve can cause impairments in extraocular movements such as upward gaze, downward gaze and adduction, which our patient did not experience.

Although physiologic anisocoria (c) occurs in $20 \%$ of the general population, the difference in size between pupils is usually less than $0.5 \mathrm{~mm}$
Competing interests: None declared.

This article has been peer reviewed.

Correspondence to:

Julio C. Furlan,

jcfurlan@gmail.com

CMAJ 2014. DOI:10.1503 /cmaj.130581
Box 1: Test for pupillary dilatation lag to show impaired pupillary dilation in paresis of the iris dilator ${ }^{1}$

- In ambient light, estimate the difference in size between pupils (anisocoria)

- Illuminate the eyes tangentially from below with a light source (e.g., flashlight) and switch off the room light.

- Observe the dilatation dynamics of both pupils carefully for 20 seconds. Photos may be taken at 5, 10, 15 and 20 seconds.

How to interpret the findings?

- In a patient with Horner syndrome, the normal pupil will dilate rapidly immediately after the room light is switched off; dilatation of the Horner pupil will be delayed because of sympathetic denervation.

- After the first few seconds of darkness, the Horner pupil will dilate slowly from the decreasing parasympathetic tone and will reach its maximal dilatation after 15-20 seconds.

- Anisocoria will be greater after 5 seconds in darkness than after 15-20 seconds.

- The presence of anisocoria with dilatation lag is highly suggestive, but not pathognomonic, of Horner syndrome. 
and not more than $1 \mathrm{~mm} .^{2}$ Also, both pupils reach maximum dilation in darkness at the same time, and the condition is not associated with ptosis, as in our patient.

Tonic pupil (d) is an idiopathic, benign cause of mydriasis that commonly affects young women between 20 and 40 years of age. ${ }^{2}$ The mydriasis is typically unilateral initially $(80 \%$ of the patients) but tends to become bilateral at a rate of $4 \%$ per year. Isolated tonic pupil does not affect eyelids.

\section{What is the next investigation?}

a. Computed tomography (CT) angiography

b. Magnetic resonance (MR) angiography

c. Doppler ultrasonography

d. Digital subtraction angiography

e. Transoral carotid ultrasonography

With acute Horner syndrome, an urgent imaging study is required to rule in or out the suspected diagnosis of carotid artery dissection. Although all of the above imaging techniques could be used to investigate carotid artery dissection, CT angiography (a), MR angiography (b) or Doppler ultrasonography (c) is traditionally chosen.,4 The first 2 are more sensitive diagnostic tools than Doppler ultrasonography and can also be used to detect intracranial extension of the carotid artery dissection, an important piece of information for planning further interventions. ${ }^{3,4}$ Given its high sensitivity and its ready availability in emergency care settings, CT angiography (a) is the most commonly used investigation in patients with suspected acute carotid artery dissection. ${ }^{3,4}$
Transoral carotid ultrasonography (e) is a relatively novel diagnostic tool that has been used to investigate distal flow in the extracranial internal carotid artery. ${ }^{5}$

In our patient, an urgent $\mathrm{CT}$ angiogram showed eccentric luminal narrowing of the distal cervical and petrous segments of the right carotid artery at the skull base, consistent with carotid artery dissection (Figure 1A). Because the dissection extended intracranially, involving the petrous portion, we started antiplatelet therapy with acetylsalicylic acid (ASA) $81 \mathrm{mg} / \mathrm{d}$. Magnetic resonance imaging and $\mathrm{MR}$ angiography of the brain and neck were done the following day to rule out progression of the arterial dissection and embolic stroke. These investigations showed an intramural hematoma compatible with the diagnosis of right internal carotid artery dissection, with extension into the cranial portion of that artery but no further extension of the dissection or ischemic stroke (Figures 1B and 1C).

Our patient was discharged home on day 3 with a prescription to continue the antiplatelet therapy. At a follow-up visit to the hospital 6 weeks later, she presented no neurologic deficits or any new cerebrovascular embolic events. The dissection was likely related to minor trauma secondary to bouts of coughing from the upper respiratory tract infection.

\section{Discussion}

\section{Horner syndrome}

Depending on the specific anatomic location of the injury or lesion in the oculosympathetic pathway, Horner syndrome is subclassified into first-
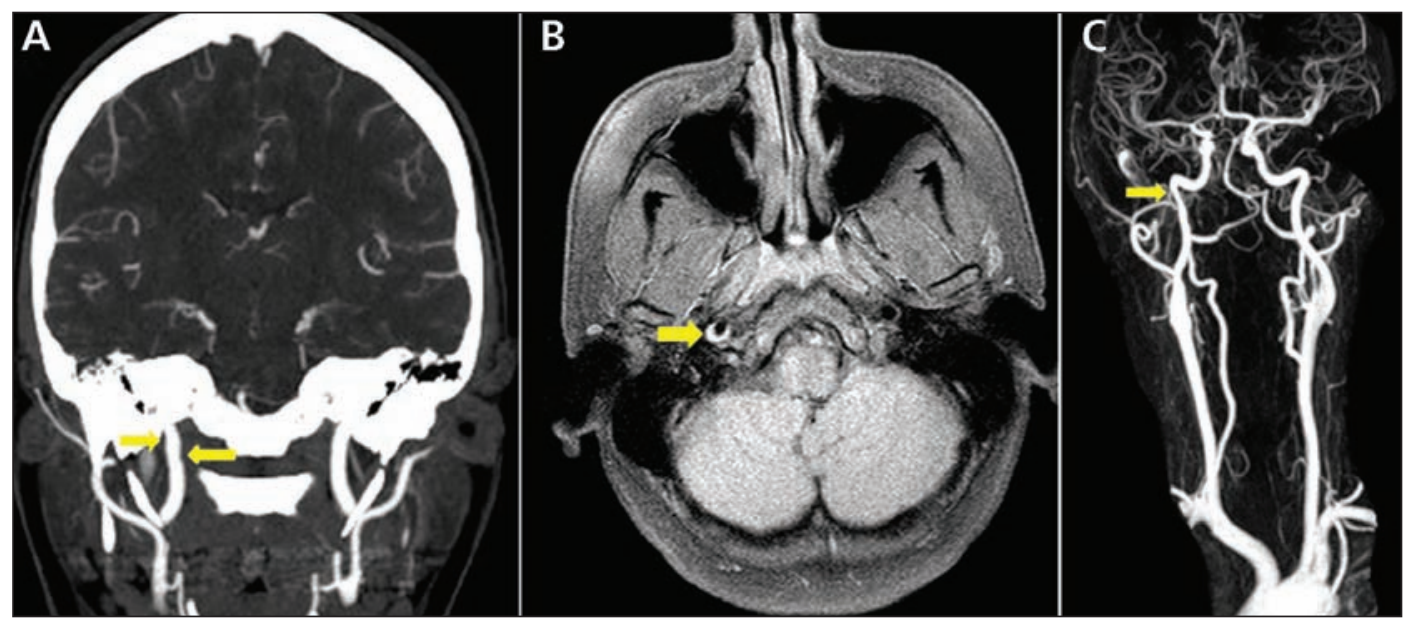

Figure 1: (A) Coronal view of a computed tomography angiogram of a 33-year-old woman with sudden onset of right-sided throbbing, severe headache and drooping of right upper eyelid. Irregularity in the right internal carotid artery is seen in the extracranial (cervical) segment above the carotid bifurcation (arrows). (B) Axial noncontrast $T_{1}$-weighted fat-saturation magnetic resonance imaging scan showing intramural hematoma (arrow) within the right internal carotid artery compatible with dissection. (C) Similar caliber change with filling defect (arrow) in the right internal carotid artery in a magnetic resonance angiogram reconstruction. 
order (central), second-order (preganglionic) and third-order (postganglionic) types (Box 2). The classic triad of ipsilateral ptosis and miosis associated with anhidrosis involving the same side of the face occurs in the first- and second-order types. It localizes the injury or lesion in the descending (first-order type) and ascending (second-order type) portions of the sympathetic nerve loop up to the superior cervical ganglion. ${ }^{2,6,7}$ When ipsilateral ptosis and miosis are seen without anhidrosis (partial Horner syndrome), a postganglionic cause should be suspected (Box 2). The lack of anhidrosis reflects the sparing of the sympathetic fibres for sweat function in the face that travel adjacent to the external carotid artery (Figure 2).

\section{Work-up of acute Horner syndrome}

Clinicians must rule out carotid artery dissection in acute Horner syndrome, typically using CT angiography, MR angiography or Doppler ultrasonography. ${ }^{7.8}$ Pathognomonic features of arterial dissection, such as an intimal flap or a double lumen, are rarely found, whereas intramural hematomas with a characteristic crescent shape adjacent to the lumen on MR angiography and CT angiography (as seen in our case) can confirm dissection. ${ }^{3}$

\section{Management of carotid dissection}

Although most patients with acute spontaneous carotid dissection are treated medically, patients presenting with subarachnoid hemorrhage or symptomatic pseudoaneurysmal dilatation may require urgent surgical intervention., ${ }^{3,7-9}$

Box 2: Types of Horner syndrome, their clinical features and common causes ${ }^{2-4}$

First order (central)

- Features: ipsilateral ptosis, miosis and anhydrosis

- Cause: hypothalamic, brainstem or spinal cord (C1 to T2) lesion or injury

Second order (preganglionic)

- Features: ipsilateral ptosis, miosis and anhydrosis

- Cause: lesion or injury involving the apex of the lung, the mediastinum or the anterior aspect of the neck

Third order (postganglionic)

- Features: ipsilateral ptosis, miosis and limited or absent anhydrosis

- Cause: lesion or injury involving the internal carotid artery, skull base, cavernous sinus, * superior orbital fissure* or orbital apex

*Sometimes accompanied by palsy of the third, fifth or sixth cranial nerve.
Despite the lack of evidence from randomized clinical trials, current guidelines suggest that use of either an anticoagulant (heparin, low-molecularweight heparin or warfarin) or an antiplatelet (ASA, clopidogrel or a combination of extendedrelease dipyridamole plus ASA) for at least 3-6 months is reasonable to treat acute extracranial carotid or vertebral arterial dissection associated with ischemic stroke or transient ischemic attack. ${ }^{10}$ Typically, pharmacologic treatment is started as soon as possible in the acute phase of the dissection. The most recent Cochrane review of antithrombotic drugs for extracranial carotid artery dissection found no randomized trials comparing either anticoagulants or antiplatelet drugs with a

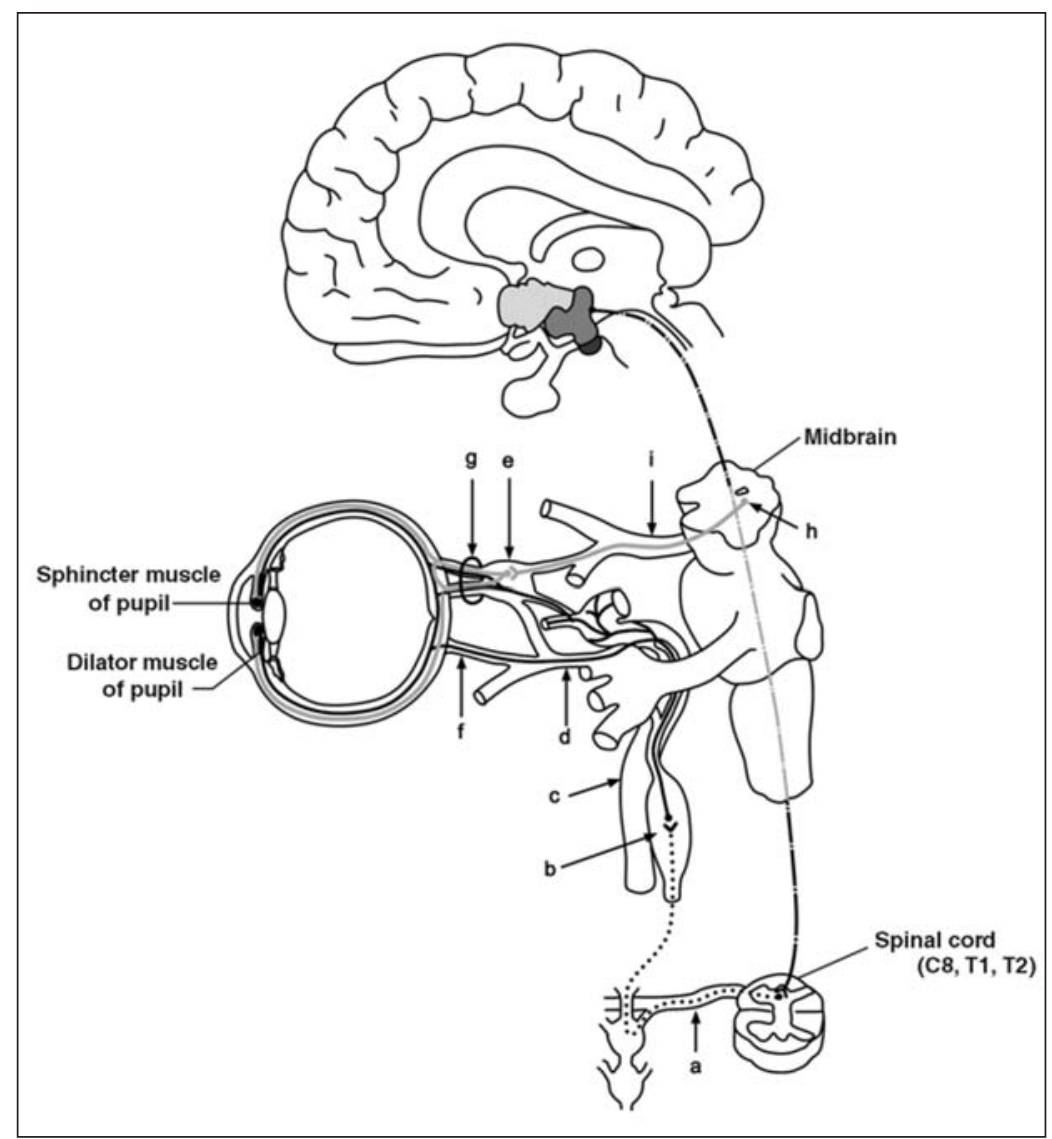

Figure 2: Anatomy of the oculosympathetic pathway divided into first-order (central), second-order (preganglionic) and third-order (postganglionic) neurons. First-order neurons (dashed line) originate from the posterolateral hypothalamus and descend into the brainstem and intermediolateral column of the spinal cord, where they synapse at the cervical (C8) and thoracic (T1-T2) levels. Second-order neurons (dotted line) exit the ventral spinal roots (a) and arch over the apex of the lung to join the cervical sympathetic chain, subsequently synapsing in the superior cervical ganglion (b). Third-order neurons (solid lines) run along the carotid artery (c) into the cavernous sinus, as well as along the ophthalmic branch (d) of the trigeminal nerve, with fibres either joining the nasociliary branch of the trigeminal nerve or passing through the ciliary ganglion (e) to reach the eye as long ( $f$ ) and short $(g)$ ciliary nerves. Sudomotor fibres to the face, but not the medial forehead, travel along the external carotid artery. Preganglionic parasympathetic fibres (grey lines) originate from the accessory oculomotor nucleus (h), exit as the oculomotor nerve (i), synapse at the ciliary ganglion and reach the eye as short ciliary nerves. Reprinted from Lee et $\mathrm{al}^{6}$ with permission from the American Journal of Roentgenology. 
control, ${ }^{9}$ nor were randomized trials found that directly compared anticoagulants and antiplatelet drugs. ${ }^{11}$ Evidence from nonrandomized studies does not favour the use of anticoagulants over antiplatelets or vice versa. ${ }^{11}$ The Cervical Artery Dissection in Stroke Study (CADISS) is an ongoing multicentre clinical trial in which patients were randomly assigned to receive antiplatelet therapy (ASA, dipyridamole or clopidogrel alone or in dual combination) or anticoagulant therapy (heparin followed by warfarin) for at least 3 months. ${ }^{12}$ A recent meta-analysis combining data from the nonrandomized arm of CADISS with results from previous nonrandomized studies failed to provide clear guidance on the relative efficacy of anticoagulation compared with antiplatelet therapy in the prevention of stroke after acute extracranial carotid and vertebral artery dissection. ${ }^{13}$

Because intracranial extension of the carotid artery dissection (as seen in our patient) increases the risk of subarachnoid hemorrhage, antiplatelet therapy tends to be preferred over anticoagulation., ${ }^{3,4}$ Still, analysis of data from a large singlecentre registry indicated that patients with intracranial artery dissection without subarachnoid hemorrhage had a favourable outcome with anticoagulants. ${ }^{14}$ To date, there is no clear evidencebased guidance on the benefits of loading with an antiplatelet agent during acute dissection, or on the benefits of switching to another platelet agent such as clopidogrel or anticoagulation in patients who are already taking ASA.

The rationale for pharmacologic treatment of acute carotid dissection is to prevent thromboembolic complications. There is no evidence to suggest that pharmacologic treatment prevents recurrence of carotid artery dissection. Expert opinion on the duration of pharmacologic therapy tends to hinge on when the luminal stenosis improves. ${ }^{4}$ One approach is to repeat either CT angiography or MR angiography after 3 months of the initial medical treatment to assess the luminal stenosis. ${ }^{8}$ The recanalization rate is high within the first 3 months after carotid dissection. If there is still evidence of luminal stenosis, another 3month period of medical treatment is usually recommended..$^{4,7}$ When anticoagulation is used in patients with acute extracranial carotid dissection, antiplatelet therapy may replace the anticoagulation after the initial 3-6 months of treatment. ${ }^{4,7}$

\section{Prognosis}

The prognosis depends on the initial neurologic deficits and extension of carotid artery dissection. ${ }^{79}$ The recurrence rate for all carotid artery dissections is about $2 \%$ within the first month and decreases to $1 \%$ annually. ${ }^{9}$ Higher recurrence rates have been reported among patients with familial disorders of connective tissue, such as fibromuscular dysplasia, Ehlers-Danlos syndrome type IV (vascular type), Marfan syndrome, cystic medial necrosis, polycystic kidney disease, $\alpha_{1}$-antitrypsin deficiency and osteogenesis imperfecta type I. ${ }^{3,8}$ However, people with connective tissue disorders account for a minority of those with spontaneous carotid artery dissection. ${ }^{3}$

The case we have described emphasizes the fact that even minor trauma secondary to bouts of coughing may result in carotid artery dissection, and it is important for clinicians to consider the diagnosis to prevent stroke.

\section{Conclusion}

Our case underscores the need to exclude carotid artery dissection when patients present with ipsilateral ptosis and miosis in the setting of ipsilateral neck and head pain even in the absence of neck manipulation, injury from a recent motor vehicle crash or other neck injuries. ${ }^{4,7}$ Doppler ultrasonography may be the most readily available test in an outpatient setting, but it is still important to pursue an urgent CT angiography (or MR angiography, if available) to confirm the diagnosis and to rule out intracranial extension of the carotid artery dissection and subarachnoid hemorrhage. Thus, if acute Horner syndrome due to carotid artery dissection is suspected, it is important to refer the patient to an emergency department for urgent imaging of the brain and neck.,4

Although either antiplatelet therapy or anticoagulation has been used in the management of extracranial carotid artery dissection, antiplatelet therapy is the preferred strategy for management of intracranial carotid artery dissection..$^{9,10}$ De spite the lack of evidence from randomized clinical trials to support this relatively common practice, either antiplatelet or anticoagulant treatment results in a generally good prognosis for patients who have acute carotid artery dissection without stroke and subarachnoid hemorrhage., ${ }^{3,4}$

\section{References}

1. Pilley SF, Thompson HS. Pupillary "dilatation lag" in Horner's syndrome. Br J Ophthalmol 1975;59:731-5.

2. Kline LB. The pupil. In: Kline LB, editor. Neuro-ophthalmology: review manual. 6th ed. Thorofare (NJ): Slack Incorporated; 2008:125-38.

3. Fusco MR, Harrigan MR. Cerebrovascular dissections - a review. Part I: spontaneous dissections. Neurosurgery 2011;68: 242-57; discussion 57.

4. Debette S, Leys D. Cervical-artery dissections: predisposing factors, diagnosis, and outcome. Lancet Neurol 2009;8:668-78.

5. Suzuki R, Koga M, Toyoda K, et al. Identification of internal carotid artery dissection by transoral carotid ultrasonography. Cerebrovasc Dis 2012;33:369-77.

6. Lee JH, Lee HK, Lee DH, et al. Neuroimaging strategies for three types of Horner syndrome with emphasis on anatomic location. AJR Am J Roentgenol 2007;188:W74-81.

7. Thanvi B, Munshi SK, Dawson SL, et al. Carotid and vertebral artery dissection syndromes. Postgrad Med J 2005;81:383-8.

8. Schievink WI. Spontaneous dissection of the carotid and vertebral arteries. N Engl J Med 2001;344:898-906. 
9. Patel RR, Adam R, Maldjian C, et al. Cervical carotid artery dissection: current review of diagnosis and treatment. Cardiol Rev 2012;20:145-52.

10. Brott TG, Halperin JL, Abbara S, et al. 2011 ASA/ACCF/AHA /AANN/AANS/ACR/ASNR/CNS/SAIP/SCAI/SIR/SNIS/SVM /SVS guideline on the management of patients with extracrania carotid and vertebral artery disease. A report of the American College of Cardiology Foundation/American Heart Association Task Force on Practice Guidelines, and the American Stroke Association, American Association of Neuroscience Nurses, American Association of Neurological Surgeons, American College of Radiology, American Society of Neuroradiology, Congress of Neurological Surgeons, Society of Atherosclerosis Imaging and Prevention, Society for Cardiovascular Angiography and Interventions, Society of Interventional Radiology, Society of NeuroInterventional Surgery, Society for Vascular Medicine, and Society for Vascular Surgery [published errata in Circulation 2011;124:e146 and 2012;126:e26]. Circulation 2011;124:e54-130.

11. Lyrer P, Engelter S. Antithrombotic drugs for carotid artery dissection. Cochrane Database Syst Rev 2010;(10):CD000255.

12. Cervical Artery Dissection in Stroke Study Trial Investigators. Antiplatelet therapy vs. anticoagulation in cervical artery dissection: rationale and design of the Cervical Artery Dissection in Stroke Study (CADISS). Int J Stroke 2007;2:292-6.

13. Kennedy F, Lanfranconi S, Hicks C, et al. Antiplatelets vs anticoagulation for dissection: CADISS nonrandomized arm and meta-analysis. Neurology 2012;79:686-9.

14. Metso TM, Metso AJ, Helenius J, et al. Prognosis and safety of anticoagulation in intracranial artery dissections in adults. Stroke 2007;38:1837-42.

Affiliations: Division of Neurology, Department of Medicine (Furlan, Sundaram), University of Toronto; Department of Genetics and Development (Furlan), Toronto
Western Research Institute, University Health Network; Lyndhurst Centre, Toronto Rehabilitation Institute (Furlan), University of Toronto; and Department of Ophthalmology and Vision Sciences (Sundaram), University of Toronto, Toronto, Ont.

Contributors: Both authors contributed substantially to the writing and revising of the article and gave final approval of the version submitted for publication.

CMAJ invites submissions to "What is your call?" Clinical details (including images) are presented with a multiple-choice question about the diagnosis. The answer and a brief discussion of the condition follow. We specifically invite submissions illustrating common or important radiographic and electrocardiographic diagnoses of appeal to a general audience. We require authors to obtain consent from the patient for publication of his or her story (form available at www.cmaj.ca/authors /checklist.shtml). Submit manuscripts online at http://mc.manuscriptcentral.com/cmaj.

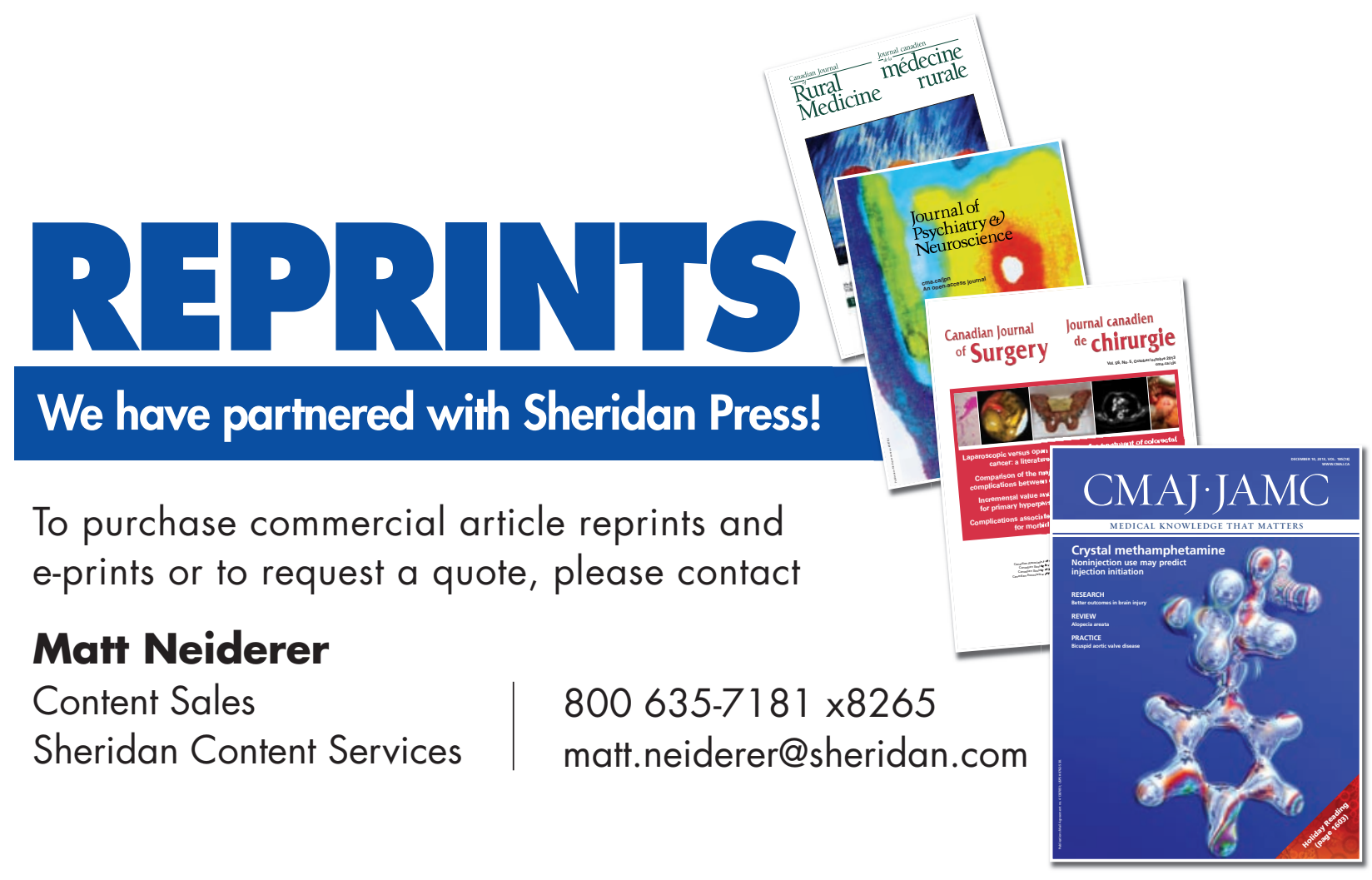

